\title{
Article
}

\section{Second mixed problem for an Euler-Poisson-Darboux equation with dirac potential}

\author{
Kaman Mondobozi Lélén ${ }^{1}{ }^{*}$, Togneme Alowou-Egnim ${ }^{1}$, Gbenouga N'gniamessan ${ }^{1}$ and Tcharie Kokou $^{1}$ \\ 1 University of Lomé, P. O. Box 1515, Togo. \\ * Correspondence: kamanlelen@gmail.com
}

Received: 18 January 2020; Accepted: 21 March 2020; Published: 31 May 2020.

Abstract: We establish the strong generalized solution of the second mixed problem for an Euler-Poisson-Darboux equation in which the free term has the form: $\gamma(t) u\left(x_{0}, t_{0}\right)$ where $u(x, t)$ is the unknown function sought at the point $\left(x_{0}, t_{0}\right)$.

Keywords: Strong generalized solution, second mixed problem, Euler-Poisson-Darboux equation, dirac potential.

MSC: 35A09, 35D35, 35L10, 35Q05.

\section{Introduction and position of the problem}

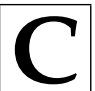

onsider the equation

$$
L_{\gamma} u \equiv L u+\gamma(t) \delta\left(x_{0}, t_{0}\right) u=f(x, t),
$$

where $u=u(x, t)$ is the unknown function defined in the domain $Q=(0, l) \times(0, T), \delta\left(x_{0}, t_{0}\right)$ is the Dirac function $\delta$ concentrated at the point $\left(x_{0}, t_{0}\right) \in Q, \delta\left(x_{0}, t_{0}\right) u=u\left(x_{0}, t_{0}\right), \gamma \in C[0, T], L=L_{0}$ is the Euler-Poisson-Darboux operator and

$$
L u \equiv \frac{\partial^{2} u(x, t)}{\partial t^{2}}+\frac{b}{t} \frac{\partial u(x, t)}{\partial t}-a^{2} \frac{\partial^{2} u(x, t)}{\partial x^{2}}, \quad b \geq 0 .
$$

With Equation (1), we associate the initial conditions:

$$
u(x, 0)=\varphi(x), \quad \frac{\partial u(x, 0)}{\partial t}=0
$$

and the boundary conditions

$$
\frac{\partial u}{\partial x}(0, t)=0, \quad \frac{\partial u(l, t)}{\partial x}=0 .
$$

It is assumed that the following conditions of conciliation of initial and boundary conditions are verified.

$$
\varphi^{\prime}(0)=0, \quad \varphi^{\prime}(l)=0 .
$$

The case in which $L$ is an ordinary differential operator and $\gamma$ a real number is treated in article [1]. Mixed problems for these so-called parabolic-type equations have been studied by Dzhenaliev, Ramazanov and Kozhanov in [2-5].

Cauchy problem and the second mixed problem for parabolic equations with Dirac potential have been studied by Baranovskaya and Yurchuk in [6]. For the Cauchy problem and the second mixed problem for the equation of form

$$
\frac{\partial^{2} u(x, t)}{\partial t^{2}}-a^{2} \frac{\partial^{2} u(x, t)}{\partial x^{2}}+\gamma \delta\left(x_{0}, t_{0}\right) u=f(x, t),
$$

where $\gamma$ is constant, were studied by Moiseev and Yurchuk in [7]. The equations of the form (5) for $\gamma=0$ and the more general equations have been studied in [8-10].

In our work, the equation contains not only the speed which is inversely proportional to time $\frac{b}{t} \frac{\partial u}{\partial t}$ but the term $\gamma(t) \delta\left(x_{0}, t_{0}\right) u$ where $\gamma(t)$ allows the control of the wave propagation at the level of the barriers in $\left(x_{0}, t_{0}\right)$. 
These terms did not exist anywhere in the equations studied by our predecessors. So our equation generalizes the equations used in the works cited above.

In the current work, we demonstrate the existence and the uniqueness of the strong generalized solution of the problem (1), (3), (4).

\section{Existence and uniqueness of the strong generalized solution of the problem (1), (3), (4).}

Let's denote by the symbol

$$
D(L)=\left\{u \in C^{2}(Q) \cup C^{1}(\bar{Q}), u(x, 0)=\varphi(x), \frac{\partial u}{\partial t}(x, 0)=\frac{\partial u}{\partial x}(0, t)=\frac{\partial u}{\partial x}(l, t)=0\right\} .
$$

From the results of [11], it follows that the second mixed problem

$$
\left\{\begin{array}{l}
L v(x, t)=f(x, t) \\
v(x, 0)=\varphi(x) \\
\frac{\partial v}{\partial t}(x, 0)=0 \\
\frac{\partial v(0, t)}{\partial x}=0 \\
\frac{\partial v(l, t)}{\partial x}=0
\end{array}\right.
$$

admits a strong generalized solution.

Consider the following norm

$$
\||u|\|=\left(\sup _{0 \leq t \leq T} \int_{0}^{l}\left[\left|\frac{\partial u}{\partial t}\right|^{2}+\left|\frac{\partial u}{\partial x}\right|^{2}+|u|^{2}\right] d x\right)^{\frac{1}{2}} .
$$

This norm defines the total energy (sum of kinetic energy and potential energy). We easily notice that if $|\|u\|| \mid<\infty$, then the function $u$ is continuous in $\bar{Q},(u \in C(\bar{Q}))$ and the value $u\left(x_{0}, t_{0}\right)$ has a meaning.

Let's set the norm in the Sobolev space

$$
W_{2}^{1}(0, l)=\left\{u \in L^{1}(0, l) / u^{\prime \prime} \in L^{1}(0, l)\right\}
$$

by the symbol

$$
\|\varphi\|_{1}=\left(\int_{0}^{l}\left[\left|\varphi^{\prime}(x)\right|^{2}+|\varphi(x)|^{2}\right] d x\right)^{\frac{1}{2}}
$$

Definition 1. We call strong generalized solution of the second mixed problem (1), (3), (4), the function $u(x, t)$ with the norm over (7), that if it's exists, the sequence $u_{n} \in D(L)$, we have the following equalities:

$$
\begin{gathered}
\left.\lim _{n \longrightarrow \infty}\left|\| u_{n}-u\right|\right|^{2}=0, \\
\lim _{n \longrightarrow \infty} \int_{Q}\left|L_{\gamma} u_{n}-f\right|^{2} d x d t=0, \\
\lim _{n \longrightarrow \infty}\left\|u_{n}(x, 0)-\varphi\right\|_{1}^{2}=0 .
\end{gathered}
$$

Remark 1. The same definition is valid for the strong generalized solution of the second mixed problem (6) when in the formula (10) instead of the operator $L_{\gamma}$, we take the operator $L$.

Theorem 1. Let $f \in L_{2}(Q), \gamma \in C[0, T], \varphi \in W_{2}^{1}(0, l)$ and

$$
\int_{0}^{t_{0}} s^{-b} \int_{0}^{s} \tau^{b} \gamma(\tau) d \tau d s \neq-1
$$


Then the second mixed problem (1), (3), (4) admits a strong generalized solution

$$
u(x, t)=v(x, t)-\frac{v\left(x_{0}, t_{0}\right) \int_{0}^{t} s^{-b} \int_{0}^{s} \tau^{b} \gamma(\tau) d \tau d s}{1+\int_{0}^{t_{0}} s^{-b} \int_{0}^{s} \tau^{b} \gamma(\tau) d \tau d s},
$$

where $v(x, t)$ is the strong generalized solution of the second mixed problem (6).

Proof. From the results of the work [11], it follows that, if $f$ belongs $L_{2}(Q), \varphi \in W_{2}^{1}(0, l)$, then $\varphi \in L_{2}(Q)$ and the second mixed problem (6) admits a strong generalized solution $v(x, t)$ with the finished norm (7). It means that there is a sequence $v_{n} \in D(L)$, as:

$$
\begin{gathered}
\left.\lim _{n \longrightarrow \infty}\left|\| v_{n}-v\right|\right|^{2}=0, \\
\lim _{n \longrightarrow \infty} \int_{Q}\left|L v_{n}-f\right|^{2} d x d t=0, \\
\lim _{n \longrightarrow \infty}\left\|v_{n}(x, 0)-\varphi\right\|_{1}^{2}=0 .
\end{gathered}
$$

The sequence $v_{n}$ can not be unique but as for the function $v(x, t)$ it must be unique.

Consider the sequence

$$
u_{n}(x, t)=v_{n}(x, t)-\frac{v_{n}\left(x_{0}, t_{0}\right) \int_{0}^{t} s^{-b} \int_{0}^{s} \tau^{b} \gamma(\tau) d \tau d s}{1+\int_{0}^{t_{0}} s^{-b} \int_{0}^{s} \tau^{b} \gamma(\tau) d \tau d s}
$$

where $v_{n}(x, t)$ is the sequence that defines the strong generalized solution $v(x, t)$ of the second mixed problem (6).

It is clear that

$$
u_{n}(x, t) \in D\left(L_{\gamma}\right)=D(L) .
$$

From the equality (14), it follows that the limit function $v(x, t)$ is continuous in $\bar{Q},(v \in C(\bar{Q}))$ and

$$
\lim _{n \longrightarrow \infty}\left|v_{n}\left(x_{0}, t_{0}\right)-v\left(x_{0}, t_{0}\right)\right|=0 .
$$

So from equality (14), from expression (17) and from equality (18), follows the equality (9) where the function $u(x, t)$ is defined by the formula (13) and $v(x, t)$ is the strong generalized solution of the second mixed problem (6).

As

$$
\begin{aligned}
L_{\gamma} u_{n} & =L_{\gamma} v_{n}-\frac{v_{n}\left(x_{0}, t_{0}\right) \gamma(t)+\gamma(t) v_{n}\left(x_{0}, t_{0}\right) \int_{0}^{t} s^{-b} \int_{0}^{s} \tau^{b} \gamma(\tau) d \tau d s}{1+\int_{0}^{t_{0}} s^{-b} \int_{0}^{s} \tau^{b} \gamma(\tau) d \tau d s} \\
& =L v_{n}+\gamma(t) v_{n}\left(x_{0}, t_{0}\right)-\frac{v_{n}\left(x_{0}, t_{0}\right) \gamma(t)+\gamma(t) v_{n}\left(x_{0}, t_{0}\right) \int_{0}^{t} s^{-b} \int_{0}^{s} \tau^{b} \gamma(\tau) d \tau d s}{1+\int_{0}^{t_{0}} s^{-b} \int_{0}^{s} \tau^{b} \gamma(\tau) d \tau d s} \\
& =L v_{n} .
\end{aligned}
$$

Then on the basis of (15), it follows from the equality (19), the equality (10). As $v_{n}(x, 0)=u_{n}(x, 0)$, then the limit (11) comes from the limit (16).

This demonstrates the existence of the strong generalized solution of the second mixed problem (1), (3), (4).

Theorem 2. Let $f \in L_{2}(Q), \gamma \in C[0, T], \varphi \in W_{2}^{1}(0, l)$ and

$$
\int_{0}^{t_{0}} s^{-1} \int_{0}^{s} \tau \gamma(\tau) d \tau d s \neq-1
$$

Then the second mixed problem (1), (3), (4) admits a unique strong generalized solution

$$
u(x, t)=v(x, t)-\frac{v\left(x_{0}, t_{0}\right) \int_{0}^{t} s^{-1} \int_{0}^{s} \tau \gamma(\tau) d \tau d s}{1+\int_{0}^{t_{0}} s^{-1} \int_{0}^{s} \tau \gamma(\tau) d \tau d s},
$$


where $v(x, t)$ is the strong generalized solution of the second mixed problem (6).

Proof. To demonstrate the uniqueness of the strong generalized solution of the problem (1), (3), (4), let's show for that if $\tilde{u}(x, t)$ is a strong generalized solution of the problem ((1)), (3), (4), then $\tilde{u}(x, t)=0$. According to the definition of strong generalized solution, there exist a sequence $\tilde{u}_{n} \in D(L)$ for which, there is the limit

$$
\begin{gathered}
\lim _{n \longrightarrow \infty}||\left|\tilde{u}_{n}-\tilde{u}\right| \|^{2}=0, \\
\lim _{n \longrightarrow \infty} \int_{Q}\left|L_{\gamma} \tilde{u}_{n}\right|^{2} d x d t=0, \\
\lim _{n \longrightarrow \infty}\left\|\tilde{u}_{n}(x, 0)\right\|_{1}^{2}=0 .
\end{gathered}
$$

From equality (21), it follows that

$$
\lim _{n \rightarrow \infty} L\left(\tilde{u}_{n}(x, t)-v_{\gamma}(x, t) \tilde{v}_{n}\left(x_{0}, t_{0}\right)=0,\right.
$$

where

$$
v_{\gamma}(x, t) \equiv v_{\gamma}(t)=\int_{0}^{t} s^{-b} \int_{0}^{s} \tau^{b} \gamma(\tau) d \tau d s .
$$

From Equations (22) and (24), it follows that

$$
\lim _{n \rightarrow \infty}\left(\tilde{u}_{n}(x, t)-v_{\gamma}(x, t) \tilde{u}_{n}\left(x_{0}, t_{0}\right)\right)=0 .
$$

From Equations (23) and (25), it follows that the function:

$$
\tilde{u}(x, t)=\tilde{u}(x, t)-v_{\gamma}(x, t) \tilde{u}\left(x_{0}, t_{0}\right)
$$

is the strong generalized solution of the homogeneous problem (5).

From the uniqueness of the strong generalized solution of the problem (5), we conclude

$$
\tilde{u}(x, t) \equiv 0 .
$$

So from the formula (26), it follows that $\tilde{u}\left(x_{0}, t_{0}\right)\left(1-v_{\gamma}\left(x_{0}, t_{0}\right)\right)=0$.

As $v_{\gamma}\left(x_{0}, t_{0}\right) \neq 1$, then $\tilde{u}\left(x_{0}, t_{0}\right)=0$.

Therefore $\tilde{u}(x, t)$ is the strong generalized solution of the homogeneous problem (5). From the uniqueness of the strong generalized solution of the homogeneous problem $(5), \tilde{u}(x, t)=0$. The theorem is thus demonstrated.

Passing to the limit in equality (17), we obtain that the strong generalized solution of the second mixed problem (1), (3), (4) is of the form:

$$
u(x, t)=v(x, t)-\frac{v\left(x_{0}, t_{0}\right) \int_{0}^{t} s^{-b} \int_{0}^{s} \tau^{b} \gamma(\tau) d \tau d s}{1+\int_{0}^{t_{0}} s^{-b} \int_{0}^{s} \tau^{b} \gamma(\tau) d \tau d s}
$$

In the particular case, when $\gamma(t)=\gamma$-constant the strong generalized solution of the second mixed problem (1), (3), (4) is of the form:

$$
u(x, t)=v(x, t)-\frac{v\left(x_{0}, t_{0}\right) \frac{1}{2(b+1)} t^{2} \gamma}{1+\frac{1}{2(b+1)} t_{0}^{2} \gamma},
$$

where $v(x, t)$ is the strong generalized solution of the second mixed problem (6).

\section{References}

[1] Nakhushev, A. M. (1983). Loaded equations and their applications. Equations Differentials, Tome 19(1), 86-94.

[2] Dzhenaliev, M. T. (2001). Loaded equations with periodic boundary conditions. Differential Equations, 37(1), 51-57.

[3] Dzhanaliev, M. T. \& Ramazanov, M. I. (2007). On a boundary problem for a spectral operator charged with heat I. Differential Equations, 43(4), 498-508. 
[4] Dzhenaliev, M. T. \& Ramazanov, M. I. (2007). On a boundary problem for a spectral operator charged with heat II. Differential Equations, 43(6), 788-794.

[5] Kozhanov, A. I. (1990). On a charged nonlinear parabolic equation and on its relation to the opposite problem. Notes Math, Tome 76(6), 84-91.

[6] Baranovskaya, S. N., \& Yurchuk, N. I. (2015). Cauchy problem and the second mixed problem for parabolic equations with the Dirac potential. Differential Equations, 51(6), 819-821.

[7] Moiseev, E. I., \& Yurchuk, N. I. (2015). Classical and generalized solutions of problems for the telegraph equation with a Dirac potential. Differential Equations, 51(10), 1330-1337.

[8] Baranovskii, F. T. (1960). A mixed problem for a degenerate hyperbolic equation. Izvestiya Vysshikh Uchebnykh Zavedenii. Matematika, 3, 30-42.

[9] Weinstein A. (1954). On the wave equation and the equation of Euler-Poisson. Proceedings of Symposia in Applied Mathematics, Wave motion and vibration theory, McGraw-Hill Book Company, New York-Toronto-London(5), 137-147.

[10] Weinstein A. (1955). The generalized radiation problem and the Euler-Poisson-Darboux equation. Summa Brasiliensis Mathematicae, Tome 3(7), 125-147.

[11] Gavrilova, N. V. \& Yurchuk, N. I. (1981). Cauchy problem for equations with differential operators of the Euler-Poisson-Darboux type. Differential Equations, Tome 17(5), 789-795. 